Trauma Berufskrankh 2008 • 10[Suppl 1]:13-15 DOI 10.1007/s10039-007-1293-5

Online publiziert: 29. November 2007

๑) Springer Medizin Verlag 2007

\author{
W. Schneiders · R. Grass · H. Zwipp \\ Klinik und Poliklinik für Unfall- und Wiederherstellungschirurgie, \\ Universitätsklinikum Dresden
}

\title{
Verletzungen der Rotatorenmanschette
}

higstellung von maximal 10 Tagen nach dem Trauma durchgeführt werden.

Zur klinischen Untersuchung gehört zunächst die Inspektion, um eventuelle Atrophien zu erkennen. Es folgt die Untersuchung des aktiven und passiven Bewegungsumfangs, wobei positive „Lagezeichen" bereits auf Defekte im Bereich der Subskapularis- (Innenrotationslagezeichen) und Infraspinatussehne (Außenrotationslagezeichen) hinweisen. Weitere Funktionstests für die Außenrotatoren sind das Dropping- und das HornblowerZeichen. Zur Beurteilung der Subskapularissehne sollten zusätzlich zum Lagezeichen der Lift-off-Test und bei Patienten mit schmerzbedingter eingeschränkter Innenrotation der Belly-Press-Test durchgeführt werden. Die Funktion der Supraspinatussehne wird mit dem Jobe-Test und dem $o^{\circ}$-Abduktions-Test geprüft.

\section{Bildgebung}

Die Röntgenuntersuchung der Schulter ist obligat. Radiologische Zeichen für eine degenerativ bedingte Rotatorenmanschettenruptur sind Sklerose im Bereich des Tuberculum majus, ein akromio-humeraler Abstand von weniger als $7 \mathrm{~mm}$, Osteophyten an der Unterkante des Akromions und Kalkablagerungen in der Rotatorenmanschette [13]. Bei eher traumatischen Rupturen zeigen sich initial radiologisch keine pathologischen Veränderungen.

Die Diagnostik sollte bei Verdacht auf einen Binnenschaden des Schultergelenks durch bildgebende Verfahren wie Ultraschall oder MRT komplettiert werden. Ausgedehnte Rupturen können klinisch sowie im Ultraschall zuverlässig diagnostiziert werden. Problematisch ist die Erkennung kleiner kompletter sowie inkompletter Rupturen. Die Sensitivität für die Detektion partieller Rupturen ist bei der Ultraschalluntersuchung deutlich geringer als bei der MRT [4, 14]. Die klinische Relevanz liegt darin, dass bei kleinen kompletten Defekten und entsprechender klinischer Symptomatik eine Rekonstruktion indiziert und meist gut möglich ist, während Partialdefekte je nach ihrer Größe und dem Anspruch des Patienten meist konservativ behandelt werden.

Die Einteilung der Rupturen erfolgt nach:

- der Lokalisation (Sektionseinteilung nach Habermeyer et al. [7]),

- der Größe (Einteilung nach Bateman [2]) und

- der Form (nach Rowe [11]: longitudinal, transversal, triangulär oder Massenruptur).

\section{Therapie}

Bezüglich der Behandlung der Rotatorenmanschettenruptur besteht in der Literatur Uneinigkeit. Einige Autoren sind der Meinung, dass - insbesondere vor dem Hintergrund, dass nur ein kleiner Teil der Rotatorenmanschette zur Zentrierung des Humeruskopfs erforderlich ist - zunächst eine konservative Therapie indiziert ist [5, $8]$. Andere fordern dagegen frühzeitig eine operative Versorgung $[1,2]$.

Bei überwiegend degenerativ bedingten Rupturen empfehlen wir immer, eine MRT-Untersuchung durchzuführen. In den Fällen, in denen bereits degenerative Veränderungen (mukoide Degenera- 


\section{Die verletzte Schulter}
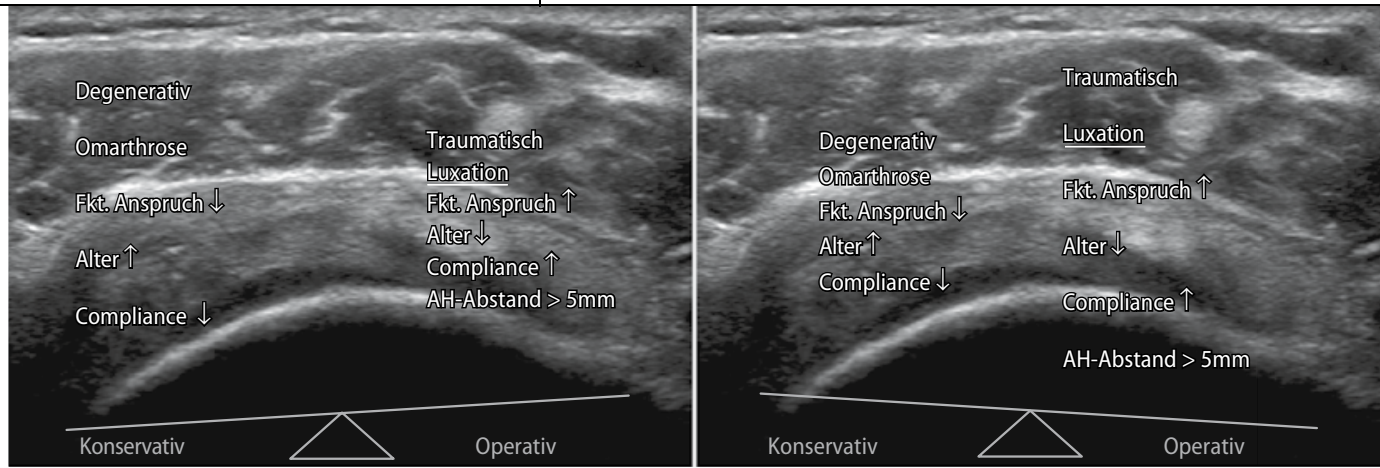

Abb. 1 Abwägung der Indikationen zur operativen bzw. konservativen Therapie bei Rotatorenmanschettenläsionen, Fkt. funktioneller, AH-Abstand akromio-humeraler Abstand
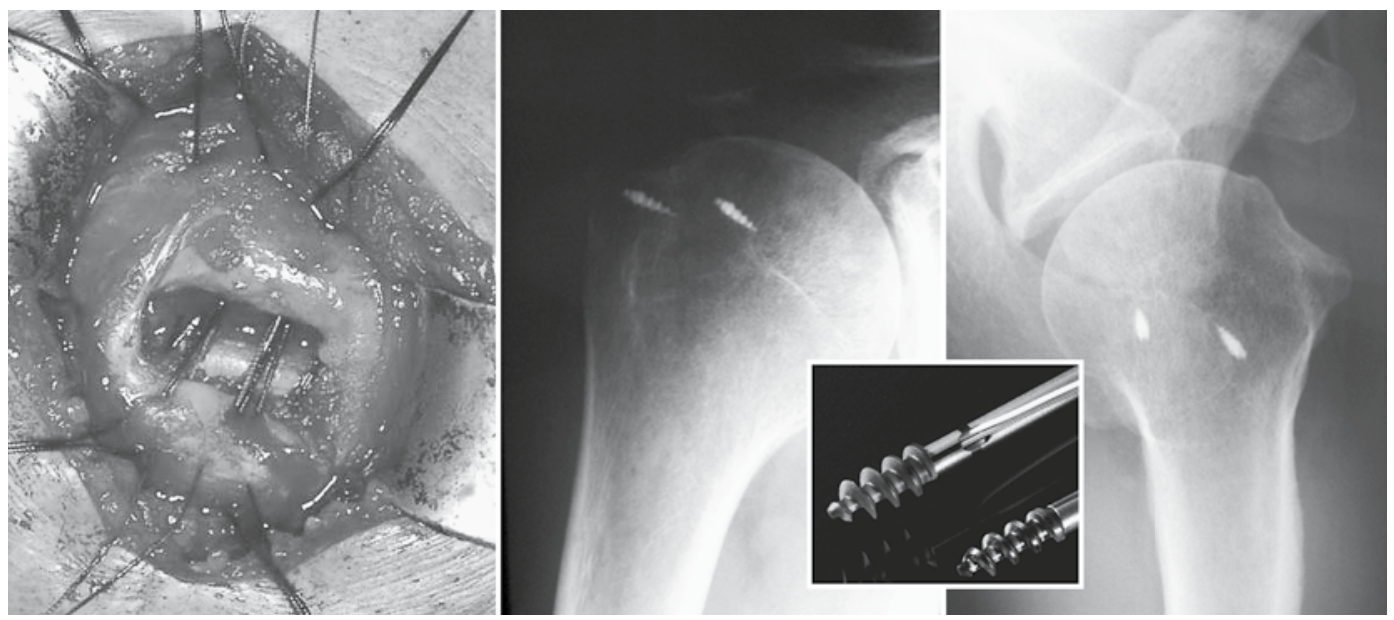

Abb. 24 Transossäre Refixation bzw. Refixation mittels Fadenanker einer ansatznahen Supraspinatussehnenruptur

tionen) im Bereich der Muskulatur der Rotatorenmanschette beschrieben sind, sollte zunächst eine konservative Therapie durchgeführt werden [6]. Sie besteht aus intensiver Physiotherapie, Verabreichung von nichtsteroidalen Antiphlogistika, Ultraschallbehandlung, Elektro-, Kryotherapie und evtl. in einer begleitenden subakromialen Infiltrationstherapie.

Indikationen für die operative Therapie sind traumatische Rupturen, $\mathrm{Zu}$ stand nach Schulterluxation und ein hoher Funktionsanspruch des Patienten. Absolute Kontraindikationen sind inaktive Patienten und Patienten ohne Compliance, begleitende Steife oder Teilsteife der Schulter, bestehende Arthritis des Schultergelenks und bestehende Omarthrose

\section{(- Abb. 1).}

Bei der operativen Therapie können die Rupturen arthroskopisch versorgt werden. Bei größeren Defekten liegen die Vorteile des offenen Vorgehens (Miniopen-Zugang) in der besseren Mobilisierbarkeit der Sehne sowie der besseren Beurteilung der Spannungsfreiheit bei deren Refixation. Ansatznahe Rupturen werden nach Anfrischen der Kortikalis am Tuberculum majus bzw. minus mittels Faden- anker oder transossären Nähten reinseriert (• Abb. 2). Zusätzlich sollte bei der Rekonstruktion der Rotatorenmanschette im Supra- bzw. Infraspinatusbereich eine subakromiale Dekompression durchgeführt werden.

Bei nicht rekonstruierbaren Rotatorenmanschettenrupturen empfehlen wir zunächst eine konservative Behandlung. Bei therapieresistenten Beschwerden sollte ein lokales Débridement, verbunden mit einer subakromialen Dekompression unter Erhaltung des korakoakromialen Bogens, durchgeführt werden. Bei Patienten mit einem hohen Funktionsanspruch kann bei entsprechender Funktionseinschränkung eine Verlagerungsoperation von M. pectoralis major oder M. latissimus dorsi erfolgen. Diese Operationen sollten jedoch in entsprechenden Zentren für die Schulterchirurgie durchgeführt werden. Bei Patienten mit erheblicher therapieresistenter Schmerzsymptomatik und zunehmender Ausbildung einer Omarthrose bei vorliegendem Massendefekt der Rotatorenmanschette ist die endoprothetische Versorgung mittels inverser Prothese indiziert [10].

\section{Fazit}

Die Therapie der Rotatorenmanschettenruptur sollte nach einem differenzierten Behandlungskonzept erfolgen. Die eher traumatischen Rupturen sollten innerhalb von 6 Wochen nach dem Unfall operativ versorgt werden. Bei Rupturen mit bereits degenerativ veränderter Muskulatur muss die Behandlung in Abhängigkeit vom Alter, der Schulterfunktion, der Compliance, den Beschwerden, dem Anspruch an die Schulterfunktion und dem Ausmaß der degenerativen Veränderungen erfolgen.

\section{Korrespondenzadresse \\ W. Schneiders}

Klinik und Poliklinik für Unfallund Wiederherstellungschirurgie, Universitätsklinikum Dresden,

Fetscherstraße 74, 01307 Dresden schneidersw@gmx.de

Interessenkonflikt. Der korrespondierende Autor gibt an, dass kein Interessenkonflikt besteht. 


\section{Literatur}

1. Bassett RW, Cofield RH (1983) Acute tears of the rotator cuff: timing of surgical repair. Clin Orthop 175: 18-24

2. Bateman JE (1963) The diagnosis and treatment of tears of the rotator cuff. Surg Clin North Am 43: 1523-1530

3. Braune C, Habermeyer P (2000) Der makroskopische Aspekt der Rotatorenmanschettenruptur bei traumatischen und atraumatischen Rupturformen. Unfallchirurg 103: 462-467

4. Bryant L, Shnier R, Bryant C et al. (2002) A comparison of clinical estimation, ultrasonography, magnetic resonance imaging, and arthroscopy in determining the size of rotator cuff tears. J Shoulder Elbow Surg 11: 219-224

5. DePalma AF (1983) Surgery of the shoulder. Saunders, Philadelphia

6. Ellman H (1990) Diagnosis and treatment of incomplete rotator cuff tears. Clin Orthop 254: 6474

7. Habermeyer P, Schiller K, Schweiberer L (1990) Rotatorenmanschette. In: Habermeyer $P$, Krueger $P$, Schweiberer L (Hrsg) Schulterchirurgie. Urban \& Schwarzenberg, München Wien Baltimore

8. McLaughlin HL (1994) Lesions of the musculotendinous cuff of the shoulder. The exposure and treatment of tears with retraction. (Classic article 1944). Clin Orthop 304: 3-4

9. Resch PP, Ritter E, Matschi W (2000) Transfer of the pectoralis major muscle for the treatment of irreparable rupture of the subscapularis tendon. J Bone Joint Surg Am 82: 372-382

10. Röhrich G, Raffl M, Seebauer L (2003) Differenzialtherapie des großen und massiven Rotatorenmanschettendefekts. Z Orthop Ihre Grenzgeb 141:

11. Rowe CR (1988) The shoulder. Churchill Livingstone, Edinburgh London New York

12. Schweiberer $L$ (1996) Schulterchirurgie. Urban \& Schwarzenberg, München Wien Baltimore

13. Südkamp NP (2001) Die Rotatorenmanschettenruptur. Zentralbl Chir 126: 177-183

14. Teefey SA, Rubin DA, Middleton WD et al. (2004) Detection and quantification of rotator cuff tears. Comparison of ultrasonographic, magnetic resonance imaging, and arthroscopic findings in seventy-one consecutive cases. J Bone Joint Surg Am 86-A: 708-716

15. Walch G (1993) Synthese sur l'epidemiologie et l'ethiologie des ruptures de la coiffe des rotateurs. J Lyonnaise Epaule 1993: 256-266
Trauma Berufskrankh 2008 - 10[Suppl 1]:13-15 DOI 10.1007/s10039-007-1293-5

(c) Springer Medizin Verlag 2007

\section{W. Schneiders $\cdot$ R. Grass $\cdot$ H. Zwipp Verletzungen der Rotatorenmanschette}

\section{Zusammenfassung}

Für die Behandlung von Rotatorenmanschettenrupturen ist es wichtig, die eher traumatischen von den degenerativ bedingten Formen zu unterscheiden und nach einem differenzierten Behandlungskonzept zu vorzugehen. Für die detaillierte Diagnose sind Anamnese, körperlicher Status und klinische Funktionsbeurteilung der Rotatorenmanschette entscheidend. Die Röntgenuntersuchung der Schulter ist obligat, bei Verdacht auf einen Binnenschaden des Schultergelenks sollte sie durch Ultraschall oder MRT ergänzt werden. Die Therapie der Rotatorenmanschettenruptur wird kontrovers diskutiert, manche befürworten eine konservative, andere die sofortige operative Behandlung. Indikationen für Letztere sind die eher traumatischen Rupturen, Zustand nach Schulterluxation und ein

\section{Injuries of the rotator cuff}

\section{Abstract}

When treatment is needed for rotator cuff tears it is important to differentiate between traumatic and degenerative forms and to proceed according to a finely varied treatment schedule. History, physical condition and clinical functional assessment of the rotator cuff are of decisive importance in the detailed diagnosis. X-Ray examination of the shoulder is mandatory and should be supplemented by ultrasound or MRI whenever internal damage of the shoulder joint is suspected. There is some controversy about the treatment of rotator cuff tears, some recommending conservative treatment and others, immediate surgery. Indications for the latter are traumatic tears, status following shoulder dislocation and desire in the patient for functional improvement. Surgical treatment is ab- hoher Funktionsanspruch des Patienten. Absolute Kontraindikationen sind inaktive Patienten, Patienten ohne Compliance, begleitende Steife/Teilsteife der Schulter, Arthritis des Schultergelenks und Omarthrose. Rupturen mit bereits degenerativ veränderter Muskulatur sollten zunächst eher konservativ behandelt werden. Bei zusätzlicher Defektarthropathie und persistierender Schmerzsymptomatik nach konservativer Behandlung ist die Implantation einer inversen Schulterprothese eine mögliche Behandlungsoption.

\section{Schlüsselwörter}

Rotatorenmanschettenläsion - Degeneration . Trauma - Differenziertes Behandlungsschema.Schmerzsymptomatik

solutely contraindicated in inactive or noncompliant patients and in patients with concomitant frozen shoulder (inckuding those with some movement in the joint), arthritis of the shoulder and omarthritis. Tears together with muscles that have already undergone degenerative alteration should initially be treated conservatively. In the presence of any additional defect caused by arthropathy and of pain persisting after conservative treatment an inverse shoulder prosthesis can be indicated.

\section{Keywords}

Rotator cuff lesion - Degeneration - Trauma . Varied treatment schedule $\cdot$ Painful symptoms 